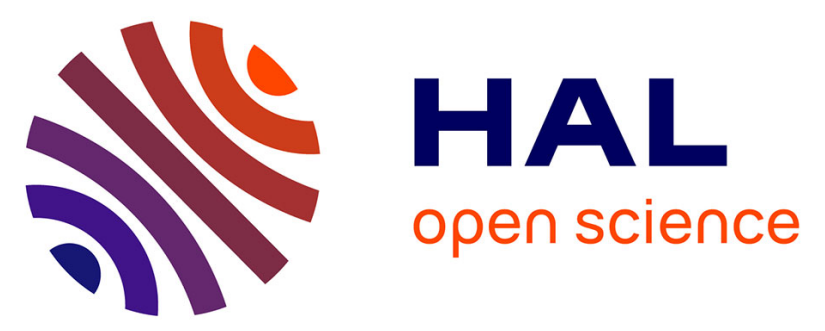

\title{
COMET 67P/CHURYUMOV-GERASIMENKO: CLOSE-UP ON DUST PARTICLE FRAGMENTS
}

M. Hilchenbach, J. Kissel, Y. Langevin, Christelle Briois, H. von Hoerner, A. Koch, R. Schulz, J. Sile'N, Kathrin Altwegg, L. Colangeli, et al.

\section{- To cite this version:}

M. Hilchenbach, J. Kissel, Y. Langevin, Christelle Briois, H. von Hoerner, et al.. COMET 67P/CHURYUMOV-GERASIMENKO: CLOSE-UP ON DUST PARTICLE FRAGMENTS. The Astrophysical journal letters, 2016, 816 (2), pp.L32. 10.3847/2041-8205/816/2/L32 . in2p3-01291758

\section{HAL Id: in2p3-01291758 \\ https://hal.in2p3.fr/in2p3-01291758}

Submitted on 10 Dec 2019

HAL is a multi-disciplinary open access archive for the deposit and dissemination of scientific research documents, whether they are published or not. The documents may come from teaching and research institutions in France or abroad, or from public or private research centers.
L'archive ouverte pluridisciplinaire HAL, est destinée au dépôt et à la diffusion de documents scientifiques de niveau recherche, publiés ou non, émanant des établissements d'enseignement et de recherche français ou étrangers, des laboratoires publics ou privés. 


\title{
COMET 67P/CHURYUMOV-GERASIMENKO: CLOSE-UP ON DUST PARTICLE FRAGMENTS
}

\author{
M. Hilchenbach ${ }^{1}$, J. Kissel ${ }^{1}$, Y. Langevin ${ }^{2}$, C. Briois ${ }^{3}$, H. von Hoerner ${ }^{4,27}$, A. Koch ${ }^{4}$, R. Schulz ${ }^{5}$, J. Silén ${ }^{6}$, K. AltwegG ${ }^{7}$, \\ L. Colangeli ${ }^{5}$, H. Cottin ${ }^{8}$, C. Engrand ${ }^{9}$, H. Fischer ${ }^{1}$, A. Glasmachers ${ }^{10}$, E. Grün ${ }^{11}$, G. Haerendel $^{12}$, H. Henkel ${ }^{4}$, \\ H. HöFner ${ }^{12}$, K. Hornung ${ }^{13}$, E. K. Jessberger ${ }^{14}$, H. Lehto ${ }^{15}$, K. Lehto ${ }^{16}$, F. Raulin ${ }^{8}$, L. Le Roy ${ }^{17}$, J. Rynö ${ }^{6}$, W. Steiger ${ }^{18}$, \\ T. Stephan $^{19}$, L. Thirkell ${ }^{3}$, R. Thomas ${ }^{3}$, K. Torkar ${ }^{20}$, K. VArmuza ${ }^{21}$, K.-P. Wanczek ${ }^{22}$, N. Altobelli ${ }^{23}$, D. Baklouti ${ }^{2}$, \\ A. Bardyn ${ }^{3,8}$, N. Fray ${ }^{8}$, H. KrüGer ${ }^{1}$, N. Ligier ${ }^{2}$, Z. Lin ${ }^{24}$, P. Martin ${ }^{3}$, S. Merouane ${ }^{1}$, F. R. Orthous-Daunay ${ }^{25}$, \\ J. Paquette ${ }^{1}$, C. Revillet ${ }^{3}$, S. Siljeström ${ }^{26}$, O. Stenzel ${ }^{1}$, and B. Zaprudin ${ }^{15}$ \\ ${ }^{1}$ Max-Planck-Institut für Sonnensystemforschung, Justus-von-Liebig-Weg 3, D-37077 Göttingen, Germany \\ ${ }^{2}$ Institut d'Astrophysique Spatiale, CNRS/Université Paris Sud, Bâtiment 121, F-91405 Orsay, France \\ ${ }^{3}$ Laboratoire de Physique et Chimie de l'Environnement et de l'Espace, CNRS/Université d'Orléans, 3 Av. de la Recherche Scientifique, F-45071 Orléans, France \\ ${ }^{4}$ von Hoerner und Sulger GmbH, Schlossplatz 8, D-68723 Schwetzingen, Germany \\ ${ }^{5}$ ESA-ESTEC, Postbus 299, 2200AG Noordwijk, The Netherlands \\ ${ }^{6}$ Finnish Meteorological Institute, Climate Research, Erik Palmenin aukio 1, P.O. Box 503, FI-00101 Helsinki, Finland \\ Physikalisches Institut, Universität Bern, Sidlerstr. 5, 3012, Bern, Switzerland \\ ${ }^{8}$ LISA, UMR CNRS 7583, Université Paris Est Créteil et Université Paris Diderot, Institut Pierre Simon Laplace, F-94000 Créteil, France \\ ${ }^{9}$ Centre de Sciences Nucléaires et de Sciences de la Matière-CSNSM, CNRS/IN2P3-Univ. Paris Sud (UMR8609), Université Paris-Saclay, \\ Bat. 104, F-91405 Orsay, France \\ ${ }^{10}$ Universität Wuppertal, FB-E, Lehrstuhl für Messtechnik, Rainer-Gruenter-Str. 21, D-42119 Wuppertal, Germany \\ ${ }^{11}$ Max-Planck-Institut für Kernphysik, Saupfercheckweg 1, D-69115 Heidelberg, Germany \\ ${ }_{13}^{12}$ Max-Planck-Institut für extraterrestrische Physik, Giessenbachstrasse, D-85748 Garching, Germany \\ ${ }_{14}^{13}$ Universität der Bundeswehr LRT-7, Werner Heisenberg Weg 39, D-85577 Neubiberg, Germany \\ ${ }^{14}$ Institut für Planetologie Universität Münster, Mail: Berghalde 31f, D-69126 Heidelberg, Germany \\ ${ }^{15}$ University of Turku, Department of Physics and Astronomy, Tuorla Observatory, Väisäläntie 20, FI-21500 Piikkiö, Finland \\ ${ }^{16}$ Laboratory of Molecular Plant Biology, Dept. of Biochemistry, PharmaCity, Itäinen Pitkäkatu 4B 6 krs, FI-20520 University of Turku, Finland \\ ${ }_{11}^{17}$ Center for Space and Habitability (CSH), University of Bern, Sidlerstrasse 5, 3012 Bern, Switzerland \\ ${ }^{18}$ RC Seibersdorf Research GmbH Business Field Aerospace Technology, A-2444 Seibersdorf, Austria \\ ${ }^{19}$ The University of Chicago, Department of the Geophysical Sciences, 5734 South Ellis Avenue, Chicago, IL 60637, USA \\ ${ }^{20}$ Space Research Institute, Austrian Academy of Sciences, Schmiedlstrasse 6, A-8042 Graz, Austria \\ ${ }^{21}$ Institute of Statistics and Mathematical Methods in Economics, Vienna University of Technology, Wiedner Hauptstrasse 7/105-6, A-1040 Vienna, Austria \\ ${ }_{22}^{22}$ Institut für Anorganische und Physikalische Chemie, Universität Bremen, Haferwende 12, D-28357 Bremen, Germany \\ ${ }^{23}$ Solar System Science Operation Division, ESA-ESAC-P.O., Box 78, E-28691 Villanueva de la Cañada, Madrid, Spain \\ ${ }^{24}$ Graduate Institute of Astronomy, NCU, No.300, Jhongda Rd., Jhongli City, Taoyuan County 32001, Taiwan
${ }^{25}$ Institut de Planétologie et d'Astrophysique de Grenoble, UMR 5274, Univ. Grenoble Alpes, CNRS, F-38000 Grenoble, France \\ ${ }^{26}$ Department of Chemistry, Materials and Surfaces, SP Technical Research Institute of Sweden, Box 857, SE-501 15 Borås, Sweden \\ Received 2015 November 18; accepted 2015 December 27; published 2016 January 12
}

\begin{abstract}
The COmetary Secondary Ion Mass Analyser instrument on board ESA's Rosetta mission has collected dust particles in the coma of comet 67P/Churyumov-Gerasimenko. During the early-orbit phase of the Rosetta mission, particles and particle agglomerates have been imaged and analyzed in the inner coma at distances between $100 \mathrm{~km}$ and $10 \mathrm{~km}$ off the cometary nucleus and at more than $3 \mathrm{AU}$ from the Sun. We identified 585 particles of more than $14 \mu \mathrm{m}$ in size. The particles are collected at low impact speeds and constitute a sample of the dust particles in the inner coma impacting and fragmenting on the targets. The sizes of the particles range from $14 \mu \mathrm{m}$ up to submillimeter sizes and the differential dust flux size distribution is fitted with a power law exponent of -3.1 . After impact, the larger particles tend to stick together, spread out or consist of single or a group of clumps, and the flocculent morphology of the fragmented particles is revealed. The elemental composition of the dust particles is heterogeneous and the particles could contain typical silicates like olivine and pyroxenes, as well as iron sulfides. The sodium to iron elemental ratio is enriched with regard to abundances in CI carbonaceous chondrites by a factor from $\sim 1.5$ to $\sim 15$. No clear evidence for organic matter has been identified. The composition and morphology of the collected dust particles appear to be similar to that of interplanetary dust particles.
\end{abstract}

Key words: comets: general - comets: individual (67P/Churyumov-Gerasimenko) - space vehicles: instruments

\section{INTRODUCTION}

The COmetary Secondary Ion Mass Analyzer (COSIMA) dust particle instrument onboard ESA mission Rosetta to comet 67P/Churyumov-Gerasimenko (hereafter 67P) exposed its first target assembly for collection of cometary dust particles at a distance of about $100 \mathrm{~km}$ from the nucleus on 2014 August 11. While the Rosetta spacecraft (Glassmeier et al. 2007) was steered closer to about $10 \mathrm{~km}$ off the nucleus center, the comet

\footnotetext{
${ }^{27}$ Deceased.
}

continued its journey into the inner solar system, from about 3.6-3.1 AU. COSIMA sequentially exposed and imaged the same target assembly on a weekly basis. Starting in early September, COSIMA analyzed particles with its time-of-flight secondary ion mass spectrometer.

\section{PARTICLE MORPHOLOGY}

COSIMA collected cometary dust particles on three porous gold targets, each $10 \mathrm{~mm} \times 10 \mathrm{~mm}$ in size and exposed simultaneously within a field of view of $15^{\circ} \times 23^{\circ}$ as described 

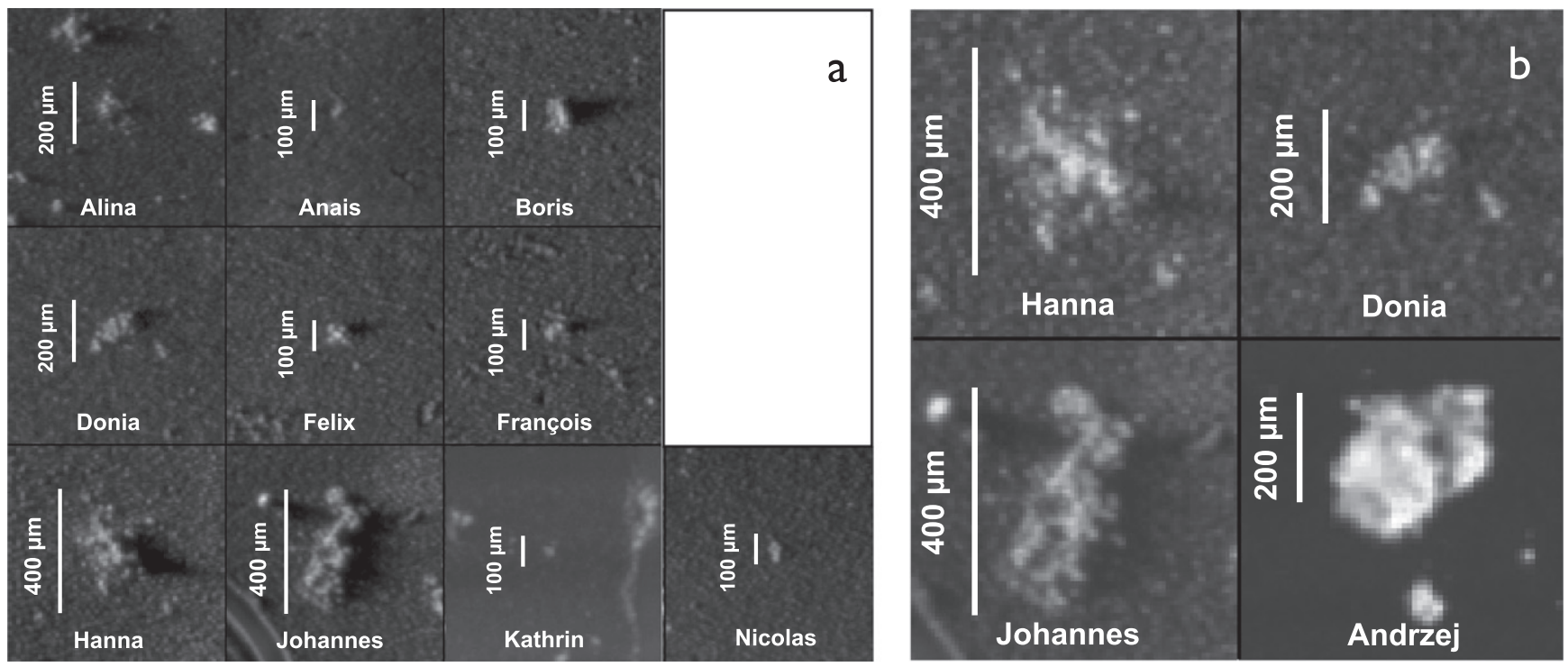

Figure 1. (a) Optical images taken by COSISCOPE of cometary particles after impacting on COSIMA gold black targets. All particles are given individual names. Grazing incidence illumination is from the left side except for Anais and the height of the particles above the target is proportional to shadows cast on the black gold target. (b) Composites of optical images obtained with left and right illumination showing the full extent of a large compact particle (Andrzej) and rubble pile and cluster agglomerates (Hanna, Donia, and Johannes).

by Kissel et al. (2007). COSIMA images the collected dust particles by means of an optical microscope (COSISCOPE). It uses two LEDs for grazing incidence illumination sequentially from left and right sides at incident angles ranging from $5^{\circ}$ to $15^{\circ}$. The dust particles are identified by light scattered by the side facing the LED against the deep black (reflectivity $<3 \%$ ) background of the porous gold. The cast shadow provides information on the height of the particle. COSISCOPE can identify grains as small as its pixel size of $14 \mu \mathrm{m}$. Identified particles are named for individual tracking and bookkeeping.

A selection of particles representing various particle types and fragmentation morphologies is shown in Figure 1(a). The particle morphologies after collection by impact are a function of the initial particle inherent material strength and impact velocity (Güttler et al. 2010; Hornung et al. 2014). The observed morphologies range from compact aggregates to rubble piles and cluster agglomerates. The particle images obtained with sub-pixel sampling (composites of four images obtained by moving the target in a square of $7 \mu \mathrm{m} \times 7 \mu \mathrm{m}$ ) hint to dust particle substructures below the nominal resolution limit of $14 \mu \mathrm{m}$. The observed particle patterns are classified in five categories: cluster agglomerates are the numerous small particles, a few tens of micrometers across, for which their sub-structure, damage or fragmentation patterns cannot be resolved, such as Anais, Francois, or Nicolas. Rubble piles tend to stick together after impact and the flocculent morphology of the fragmented particles is revealed, such as Boris, Donia, Felix, and Hanna. Shattered cluster agglomerates spread out on impact like a pancake and do not cast a long shadow, such as Alina. Glued cluster agglomerates consist of larger and smaller clumps, potentially including several holes or pits, such as Johannes. For the particles with imprints on the target larger than $100 \mu \mathrm{m}$, the ones classified as rubble piles are the dominating particle type with four detections, followed by shattered cluster, glued cluster and compact with one detection each. The particle fragment differential size distributions after impact can be fitted with power law exponents between -3.5 and -1.5 , the spread being due to the distributions of impact velocities and particle material strengths (Sator et al. 2008).

Images with both left and right illuminations added up by linear digital image processing are shown in Figure 1(b) for the larger particles Hanna, Donia, Johannes, and Andrzej. Andrzej is representative of the compact particle class, which may have split on impact but does not show a fragmentation pattern such as Hanna or Johannes. For Andrzej and Johannes, the nonfragmented parts show visible substructures and porosity.

\section{PARTICLE SIZE DISTRIBUTION AND PARTICLE FLUX IN THE INNER COMA}

The Rosetta spacecraft was pointing most of the time toward the center of the nucleus of 67P, with less than $5^{\circ}$ off-pointing. Starting in early September, COSIMA analyzed identified particles with its time-of-flight secondary ion mass spectrometer. Intervals of up to two days were devoted to chemical analysis, and no dust was collected as no target assembly was exposed during that time. The average dust flux observed by COSIMA during each of the 11 exposures (each with an effective duration of 5-7 days) was evaluated from the number of additional particles identified on the images after the exposure when compared to the images before exposure.

The particle flux observed in the inner coma is shown in Figure 2. The particles sizes range from 15 to $225 \mu \mathrm{m}$ equivalent diameter $s$, defined as the diameter of a circle covering the same area as derived from the measured area covered by a particle or particle cluster. The particle differential size distribution $N(s) d s \sim s^{-a}$ ds is fitted by a mean power law exponent a of -3.1 , within a range of -3.7 and -1.4 (Figure 2(a)).

The dust particle flux in the $15-225 \mu \mathrm{m}$ size range versus comet distance is derived from the COSISCOPE sequential imaging time sequences binned for comet nucleus distance of Rosetta. The particle flux is a function of comet rotation, activity, spacecraft pointing, phase angle, distance to comet nucleus $d_{\mathrm{c}}$, and distance to the Sun $D_{\mathrm{S}}$. The observed particle 

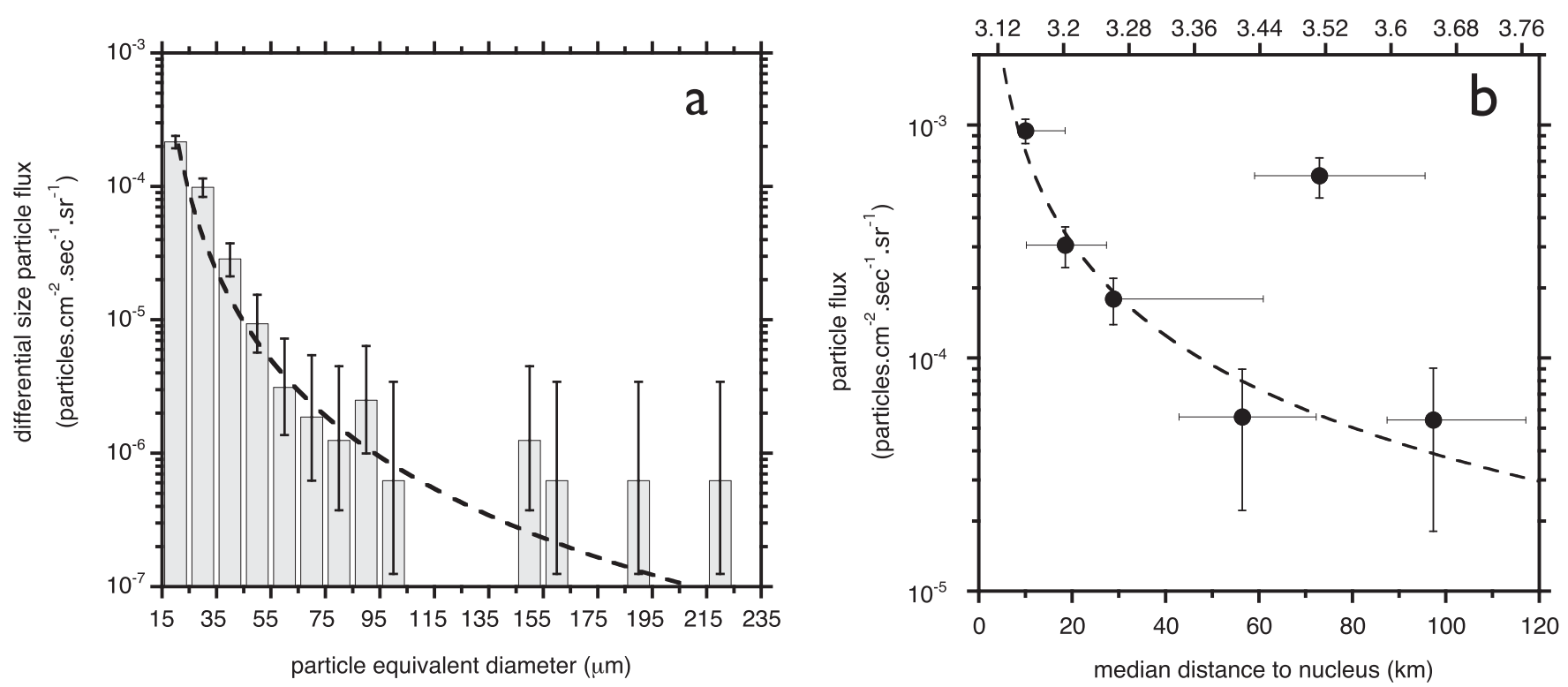

Figure 2. (a) Differential size distribution of the dust particle flux collected during the time interval 2014 August 11 to October 24 on the COSIMA gold targets. Size bins of $10 \mu \mathrm{m}$ refer to the particle equivalent diameter as derived from the COSIMA microscope images from the area covered by single particles and particle clusters. The dashed line indicates the best power law fit with an index of -3.1 . (b) Particle fluxes plotted vs. the spacecraft distance to the nucleus. The respective solar distance is indicated on the upper X-axis. The particle equivalent diameters are between 15 and $225 \mu \mathrm{m}$. The dashed line indicates the power law fit. The flux errors refer to $95 \%$ confidence levels and the bars indicate the nucleus distance intervals.

flux increases as Rosetta draws nearer to $67 \mathrm{P}$ and closer to the Sun, with one outlier at a distance of $\sim 75 \mathrm{~km}$ from the comet (Figure 2(b)). The particle flux is decreasing as

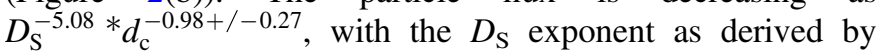
Agarwal et al. (2007) and the $d_{\mathrm{c}}$ dependence fitted by a power law function. The observed flux decreases with increasing nucleus and solar distance. The outlier with high dust flux at $d_{\mathrm{c}}=75 \mathrm{~km}$ off the nucleus might be explained by particle fragmentation close to the Rosetta spacecraft, generating a close-by dust particle source (Fulle et al. 2015).

\section{TOF-SIMS ANALYSIS AND COMPOSITION OF DUST PARTICLES}

The dust particle analysis is carried out by time-of-flight secondary ion mass spectrometry (TOF-SIMS), a technique dedicated to analyze the outermost surface layer of a solid sample. The mass resolution $m / \Delta m$ of COSIMA is about 1400 at half peak maximum at $m / z=100 \mathrm{u}$, thus allowing distinguishing elements from organic molecules around the same integer mass for $m / z<100 \mathrm{u}$. The footprint of the primary ion beam is $35 \times 50 \mu \mathrm{m}^{2}$ (full width at half maximum), therefore, depending on the size of the sampled location, individual mass spectra could contain secondary ions from the cometary particle, the target surface, or both. Mass spectra are normalized to hydrogen and spectra taken outside the particles on the target background are subtracted to remove the contribution of the secondary ions originating from the Au target within the primary ion beam footprint.

After collection on the targets, the particles can be physically altered during TOF-SIMS analysis: particles or parts of them can be lifted by electrostatic forces induced by the applied electric field of up to $1.5 \mathrm{kV} \mathrm{mm}^{-1}$ (Kok \& Renno 2006). An example is shown in Figure 3(a) for Donia. In the first image taken after the particle collection, particle Donia casts two significant shadows of 240 and $440 \mu \mathrm{m}$ length, indicating particle heights of 30 and $60 \mu \mathrm{m}$. After the first TOF-SIMS measurements the long shadow was not visible anymore and the part of the particle casting it was lost during the analysis. The other shadow shrank by $40 \mu \mathrm{m}$, and, after the second set of TOF-SIMS analyses, the particle elevation was reduced even further to $\sim 20 \mu \mathrm{m}$.

The surfaces of the particles exposed to the TOF-SIMS primary ion beam are freshly broken on impact on the COSIMA collection targets. For particle Donia, the statistically most significant peaks in the TOF-SIMS spectra in the low mass range are at $m / z=22.99 \mathrm{u}, \mathrm{Na}^{+} ; 73.05 \mathrm{u}, \mathrm{Si}\left(\mathrm{CH}_{3}\right)_{3}^{+}$a polydimethylsiloxane (PDMS) cation which is a target surface contaminant; and $114.90 \mathrm{u}, \mathrm{In}^{+}$, the primary ion. Rock forming elements such as $\mathrm{Mg}$ and $\mathrm{Fe}$ are also present in particle Donia (Figure 3(b)). In the higher mass range, an organic compound is detected at $m / z=228.25 \mathrm{u}$ which is not related to PDMS. The particle position from the optical image coincides with maximum positive secondary ion intensity for $\mathrm{Na}^{+}, \mathrm{Mg}^{+}$, and $\mathrm{Fe}^{+}$, and minimum ion intensities for PDMS, $\mathrm{Au}^{+}$, and the organic mass peak at $m / z=228.25 \mathrm{u}$ (Figure 3(c)). Detection of enhanced $\mathrm{Na}^{+}$signal as a tracer for cometary particles collected by COSIMA has been reported by Schulz et al. (2015).

The element abundances for nine analyzed particles are shown in Figure 4, normalized to $\mathrm{Fe}$ and to the abundances in CI carbonaceous chondrites according to Lodders (2010). One further analyzed particle, Anais, does not contain a detectable amount of $\mathrm{Fe}$ and is not included in Figure 4. The $\mathrm{Fe}$ normalization was used due to the uncertainty in the ion counts for $\mathrm{Si}^{+}$that could contain a contribution from PDMS even after background subtraction. Quantification was made using the relative sensitivity factors determined by analysis of series of relevant minerals by the reference model of COSIMA (Krüger et al. 2015). The composition of the ten particles analyzed is not related to the particle size or morphology after impact. For the analyzed particles, three main groups can be defined so far: 
a
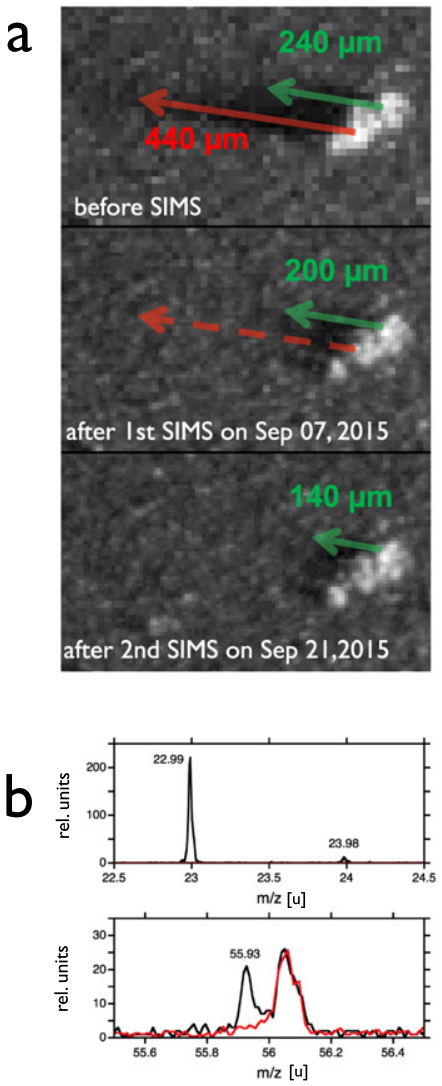

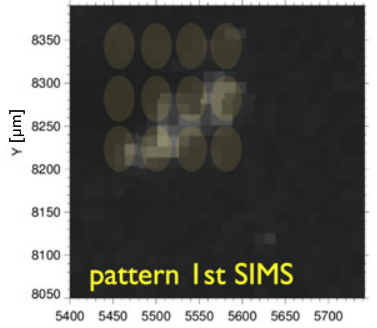

$\mathrm{x}[\mu \mathrm{m}]$

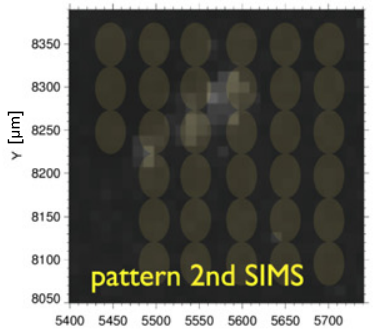

$x[\mu \mathrm{m}]$
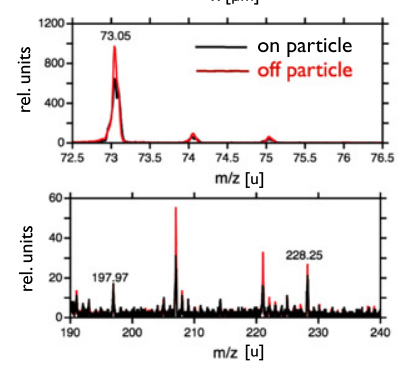

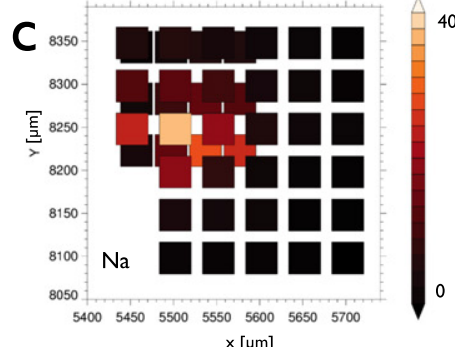

$x[\mu \mathrm{m}]$

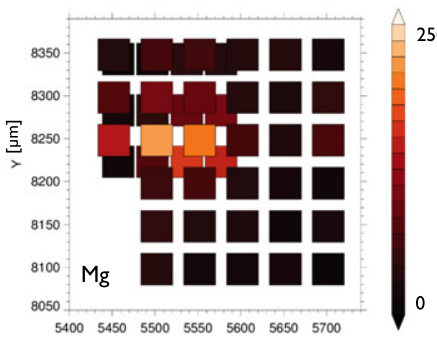

$x[\mu \mathrm{m}]$

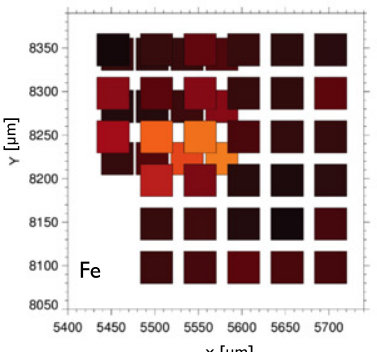

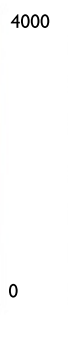
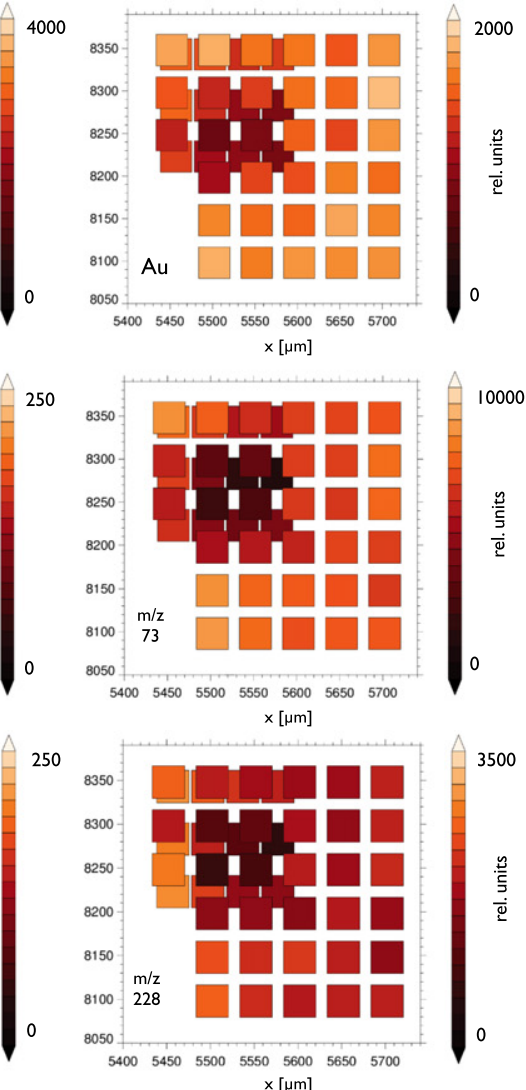

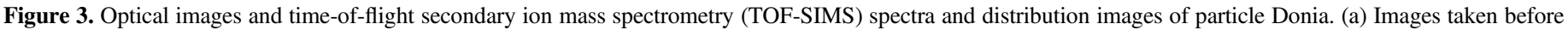

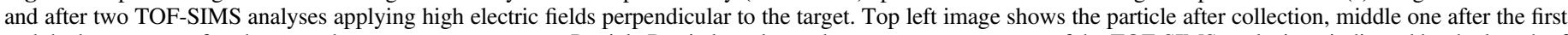

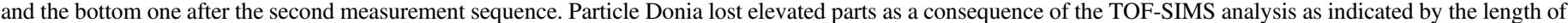

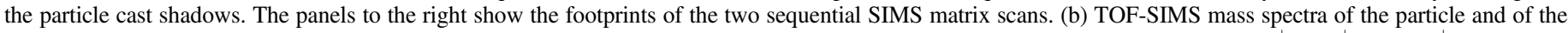

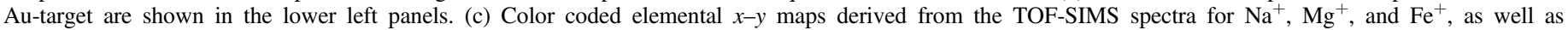
$m / z=73.05 \mathrm{u}(\mathrm{PDMS}), \mathrm{Au}^{+}$, and $m / z=228.25 \mathrm{u}$.

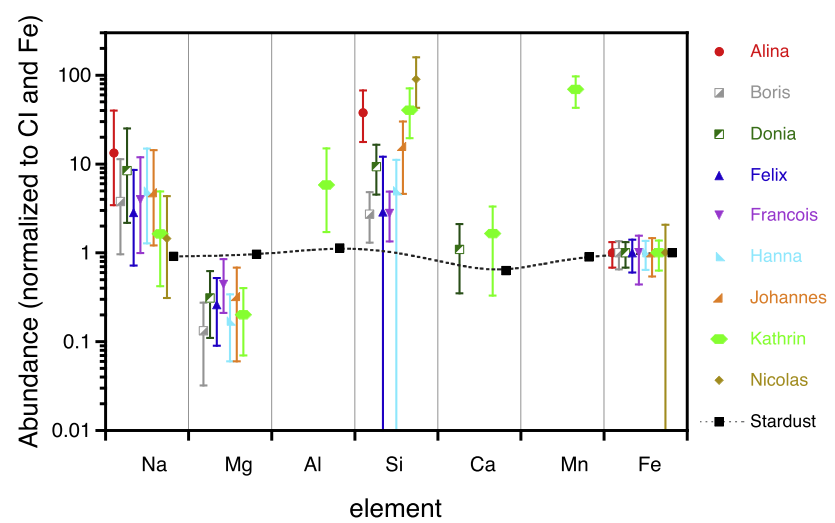

Figure 4. Relative elemental abundance, normalized to Fe and CI abundance. $\mathrm{Na}$ and $\mathrm{Si}$ are enriched in all particles and $\mathrm{Al}$ and $\mathrm{Mn}$ for one particle, Kathrin, compared to CI elemental abundance (Lodders 2010). $\mathrm{Mg}$ is depleted in all particles. The error stated refers to $95 \%$ confidence level for the statistical error, $30 \%$ assumed systematic error of TOF-SIMS calibration, and takes into account the uncertainty related to the relative sensitivity factors used for quantification (Krüger et al. 2015). For comparison, the Stardust results from comet $81 \mathrm{P} /$ Wild cometary particle analysis are shown (Ishii et al. 2008; Lanzirotti et al. 2008; Leroux et al. 2008; Stephan 2008; Stephan et al. 2008; Brownlee 2014).

Alina and Nicolas contain only $\mathrm{Na}, \mathrm{Si}$, and Fe. Boris, Donia, Felix, François, Hanna, and Johannes contain $\mathrm{Na}, \mathrm{Mg}, \mathrm{Si}$, and $\mathrm{Fe}$, with additional $\mathrm{Ca}$ for Donia. Anais and Kathrin are outliers
- Anais contains $\mathrm{Na}, \mathrm{Mg}, \mathrm{Si}$, and $\mathrm{Ca}$ (no $\mathrm{Fe}$ ), Kathrin contains $\mathrm{Na}, \mathrm{Mg}, \mathrm{Al}, \mathrm{Si}, \mathrm{Ca}, \mathrm{Mn}$, and $\mathrm{Fe}$.

For all particles, the $\mathrm{Na} / \mathrm{Fe}$ ratio is enriched with regard to $\mathrm{CI}$ by a factor from $\sim 1.5$ to $\sim 15$. $\mathrm{Na}$ is correlated to $\mathrm{Mg}$ and $\mathrm{Fe}$, but not to $\mathrm{Si}$. The host phase of $\mathrm{Na}$ is still unidentified, but our results do not support $\mathrm{Na}$ being present in $67 \mathrm{P}$ in the form of a silicate. For $\mathrm{Mg}$-bearing particles, the $\mathrm{Mg} / \mathrm{Fe}$ elemental ratio varies between $\sim 0.2$ and $\sim 0.5$. These particles could contain typical silicates like olivine and pyroxenes, as well as iron sulfides. The systematic overabundance of Fe with regard to CI abundance $(\mathrm{Mg} / \mathrm{Fe}(\mathrm{CI})=1.18)$ may be explained by a larger abundance of iron sulfides in 67P than in CI material. This is particularly clear for Alina and Nicolas, which do not contain $\mathrm{Mg}$, and could be dominated by iron sulfides. On the other hand, Anais contains no $\mathrm{Fe}$, has an atomic $\mathrm{Ca} / \mathrm{Mg}$ ratio $\sim 2$ and could be made of $\mathrm{Mg}$ and $\mathrm{Ca}$-rich silicates. Kathrin shows a complex composition, with both $\mathrm{Al}$ and $\mathrm{Ca}$ enrichment, as well as extreme $\mathrm{Mn} / \mathrm{Fe}$ ratio $(\mathrm{Mn} / \mathrm{Fe} \sim 70 \times \mathrm{CI})$. This particle could contain refractory compounds explaining the elevated atomic ratios $\mathrm{Ca} / \mathrm{Mg} \sim 0.5$ and $\mathrm{Al} / \mathrm{Mg} \sim 2.5$. The $\mathrm{Mn}$ enrichment could possibly be explained by the presence of Brownleeite $(\mathrm{MnSi})$, a mineral recently discovered in an interplanetary dust particle (IDP) of possible cometary origin (Nakamura-Messenger et al. 2010).

The organic ion mass peak at $\mathrm{m} / \mathrm{z}=228.25 \mathrm{u}$ correlates with the mass peaks at $m / z=18.04 \mathrm{u}, 30.04 \mathrm{u}, 44.05 \mathrm{u}, 58.07 \mathrm{u}$, $113.11 \mathrm{u}, 155.15 \mathrm{u}, 182.19 \mathrm{u}, 214.24 \mathrm{u}, 228.25 \mathrm{u}, 242.27 \mathrm{u}$, and 
$256.28 \mathrm{u}$. The series of mass peaks $\mathrm{m} / \mathrm{z}=214.24 \mathrm{u}$ to $256.28 \mathrm{u}$ is correlated with low mass peak series $18.04 \mathrm{u}$ to $58.07 \mathrm{u}$ indicating that these peaks could originate from compounds that contain amine functional groups. The mass difference pattern of $14.02 \mathrm{u}\left(\mathrm{CH}_{2}\right)$ is consistent with aliphatic amine. The high mass defect for the series of peak points toward highly saturated compounds. Despite a good mass match at $m / z=155.15 \mathrm{u}$ with methylated hexamethylenetetramine [Me$\mathrm{HMT}+\mathrm{H}]^{+}$, a molecule often considered as a signature of UV and thermal processing of icy mixture simulating in the laboratory interstellar and cometary ice chemistry (Muñoz Caro et al. 2004; Vinogradoff et al. 2013), the lack of correlation with the expected ion fragments at $m / z=42.03 \mathrm{u}\left(\mathrm{C}_{2} \mathrm{H}_{4} \mathrm{~N}^{+}\right)$ rules out this assignment (Le Roy et al. 2015). So far a cometary origin of all of these peaks is doubtful as those peaks are also detected in mass spectra taken before the first exposure of the targets.

\section{DISCUSSION}

The particle morphologies and the diversity in morphology of the collected particles and particle fragments shown in Figure 1 point toward highly heterogeneous families of particles. These are reminiscent of cosmic dust particles collected on Earth: stratospheric IDPs collected by NASA (Bradley 2014; Stodolna et al. 2014) and micrometeorites collected in polar snow (Duprat et al. 2007; Dobrica et al. 2009). The compact particles could be related to the "terminal particles," which survived impact at $6 \mathrm{~km} \mathrm{~s}^{-1}$ in the Stardust aerogel targets (Brownlee et al. 2006), or they are more similar in morphology and inner structure to the other identified particle classes, but have impacted with a lower velocity, and therefore, no apparent fragmentation could be identified in the COSICOPE images.

The particle flux size distribution with a mean power law exponent of -3.1 is a bit steeper than observed by the Rosetta dust detector GIADA for larger particle sizes while the lack of observation of craters implies particle impact speeds $<50 \mathrm{~m} \mathrm{~s}^{-1}$, compatible with speeds $<10 \mathrm{~m} \mathrm{~s}^{-1}$ as determined by GIADA (Rotundi et al. 2015). The difference for the size distribution functions might be due to the particle flux of the smaller particles detected only by COSIMA and the observed COSIMA particle flux size distribution might be better fitted with a more complex size frequency distribution: steeper for the small and shallower for larger particles. The mean particle size distributions observed in the dust tails being steeper than in the coma would be in line with this interpretation if the small particles observed next to the comet would be the source population of small particles observed remotely in the dust tail (Fulle et al. 2004; Tuzzolino et al. 2004).

The power law exponent fitted for the dependence of the particle flux on the nucleus distance $d_{\mathrm{c}}$ indicates a linear decrease of the dust flux of particles with respect to the comet distance during Rosetta's close approach to the nucleus. A possible explanation would be the continuous fragmentation of large parent dust particles (Clark 2004). These travel off the nucleus with their flux decreasing with $d_{\mathrm{c}}^{-2}$. Integration of such an extended "parent" dust source would result in a linear decrease of the particle flux observed by COSIMA for particles with constant velocity. But the observed particle velocity is increasing with the nucleus distance (Della Corte et al. 2015), giving rise to an increased particle flux due to higher particle velocity and not higher particle density. Even very close to the comet nucleus, COSIMA collected small particles and no size threshold is observed in the flux of particles ejected off 67P's surface. Models by Skorov \& Blum (2012) and Blum et al. (2015) predict a minimum and maximum particle size for the particle ejection off the dust mantle driven by water sublimation. COSIMA observations are in accordance with these models if the small particles are lifted off with the large particles and travel faster than these, as they pass through the acceleration region.

The elemental composition of the dust particles is heterogeneous as it has been described for 1P/Halley based on the Giotto and Vega mission data (Kissel et al. 1986a, 1986b; Langevin et al. 1987; Jessberger et al. 1988). With the possible exception of elevated $\mathrm{Na}$ contents, the elementary composition of 67P-CG particles is broadly compatible with the inorganic compositions of ultracarbonaceous Antarctic micrometeorites (UCAMMs, Dobrica et al. 2012), carbonaceous chondrites, and Wild 2 samples returned by the Stardust mission (Flynn et al. 2006; Zolensky et al. 2006; Frank et al. 2014).

The cometary particles collected and measured until 2014 October 24, do not display clear signatures of cometary organic material in the TOF-SIMS analysis. This could be due to the presence of macromolecular organic material that have a very weak signature in TOF-SIMS spectra. The organic molecules could therefore be similar to macromolecular insoluble organic matter as extracted from carbonaceous meteorites (Cody \& Alexander 2005; Derenne \& Robert 2010), or making up the bulk of UCAMMs (Duprat et al. 2010; Dartois et al. 2013), or resembling some irradiated ice laboratory analogs like hydrogenated amorphous carbons (Jenniskens et al. 1993; Dartois \& Muñoz Caro 2007). No sign of small organic molecules such as amino acids, nucleobases, carboxylic acid, hydrocarbons (aliphatic or aromatics), like observed in the soluble fraction extracted from carbonaceous meteorites or Stardust mission (Botta \& Bada 2002; De Gregorio et al. 2011), were observed so far.

Both the textures of particles collected at low impact speeds and the compositions of 67P particles analyzed by COSIMA suggest a link with extraterrestrial dust particles collected on Earth such as IDPs and micrometeorites.

COSIMA was built by a consortium led by the Max-PlanckInstitut für Extraterrestrische Physik, Garching, Germany in collaboration with Laboratoire de Physique et Chimie de l'Environnement et de l'Espace, Orléans, France, Institut d'Astrophysique Spatiale, CNRS/Université Paris Sud, Orsay, France, Finnish Meteorological Institute, Helsinki, Finland, Universität Wuppertal, Wuppertal, Germany, von Hoerner und Sulger GmbH, Schwetzingen, Germany, Universität der Bundeswehr, Neubiberg, Germany, Institut für Physik, Forschungszentrum Seibersdorf, Seibersdorf, Austria, Space Research Institute, Austrian Academy of Sciences, Graz, Austria and is led by the Max-Planck-Institut für Sonnensystemforschung, Göttingen, Germany. The support of the national funding agencies of Germany (DLR, grant $50 \mathrm{QP}$ 1302), France (CNES), Austria, Finland and the ESA Technical Directorate is gratefully acknowledged. We thank the Rosetta Science Ground Segment at ESAC, the Rosetta Mission Operations Centre at ESOC and the Rosetta Project at ESTEC for their outstanding work enabling the Science return of the Rosetta Mission. Rosetta is an ESA mission with contributions 
from its Member States and NASA. We thank the referee for having greatly improved the manuscript.

\section{REFERENCES}

Agarwal, J., Müller, M., \& Grün, E. 2007, SSRv, 128, 79

Blum, J., Gundlach, B., Mühle, S., \& Trigo-Rodríguez, J. M. 2015, Icar, 248, 135 Botta, O., \& Bada, J. L. 2002, SGeo, 23, 411

Bradley, J. P. 2014, in Treatise on Geochemistry, Vol. 1, ed. A. M. Davis (2nd ed.; Amsterdam: Elsevier-Pergamon), 287

Brownlee, D. 2014, AREPS, 42, 179

Brownlee, D., Tsou, P., Aléon, J., et al. 2006, Sci, 314, 1711

Clark, B. C. 2004, JGR, 109, E12S03

Cody, G. D., \& Alexander, C. M. O. D. 2005, GeCoA, 69, 1085

Dartois, E., Engrand, C., Brunetto, R., et al. 2013, Icar, 224, 243

Dartois, E., \& Muñoz Caro, G. M. 2007, A\&A, 476, 1235

De Gregorio, B. T., Stroud, R. M., Cody, G. D., et al. 2011, M\&PS, 46, 1376

Della Corte, V., Rotundi, A., Fulle, M., et al. 2015, A\&A, 583, A13

Derenne, S., \& Robert, F. 2010, M\&PS, 45, 1461

Dobrica, E., Engrand, C., Duprat, J., et al. 2009, M\&PS, 44, 1643

Dobrica, E., Engrand, C., Leroux, H., et al. 2012, GeCoA, 76, 68

Duprat, J., Dobrica, E., Engrand, C., et al. 2007, AdSpR, 39, 605

Duprat, J., Engrand, C., Maurette, M., et al. 2010, Sci, 328, 742

Flynn, G. J., Bleuet, P., Borg, J., et al. 2006, Sci, 314, 1731

Frank, D. R., Zolensk, M. E., \& Le, L. 2014, GeCoA, 142, 240

Fulle, M., Barbieri, C., Cremonese, G., \& Rauer, H. 2004, A\&A, 422, 357

Fulle, M., Corte, V. D., Rotundi, A., et al. 2015, ApJL, 802, L12

Glassmeier, K.-H., Boehnhardt, H., Koschny, D., et al. 2007, SSRv, 128, 1

Güttler, C., Blum, J., Zsom, A., Ormel, C. W., \& Dullemond, C. P. 2010, A\&A, 513, A56
Hornung, K., Kissel, J., Fischer, H., et al. 2014, P\&SS, 103, 309 Ishii, H. A., Brennan, S., Bradley, J. P., et al. 2008, M\&PS, 43, 215 Jenniskens, P., Baratta, G. A., Kouchi, A., et al. 1993, A\&A, 273, 583 Jessberger, E. K., Christoforidis, A., \& Kissel, J. 1988, Natur, 332, 691 Kissel, J., Altwegg, K., Clark, B. C., et al. 2007, SSRv, 128, 823

Kissel, J., Brownlee, D. E., Buchler, K., et al. 1986a, Natur, 321, 336

Kissel, J., Sagdeev, R. Z., Bertaux, J. L., et al. 1986b, Natur, 321, 280

Kok, J. F., \& Renno, N. O. 2006, GeoRL, 33, L19S10

Krüger, H., Stephan, T., Engrand, C., et al. 2015, P\&SS, 117, 35

Langevin, Y., Kissel, J., Bertaux, J. L., \& Chassefiere, E. 1987, A\&A, 187,761

Lanzirotti, A., Sutton, S. R., Flynn, G. J., et al. 2008, M\&PS, 43, 187

Leroux, H., Rietmeijer, F. J. M., Velbel, M. A., et al. 2008, M\&PS, 43, 97

Le Roy, L., Bardyn, A., Briois, C., et al. 2015, P\&SS, 105, 1

Lodders, K. 2010, in Principles and Perspectives in Cosmochemistry, ed. A. Goswami, \& B. E. Reddy (Berlin: Springer), 379

Muñoz Caro, G. M., Meierhenrich, U., Schutte, W. A., et al. 2004, A\&A, 413, 209

Nakamura-Messenger, K., Keller, L. P., Clemett, S. J., et al. 2010, AmMin, 95,221

Rotundi, A., Sierks, H., Corte, D. V., et al. 2015, Sci, 347, 6220

Sator, N., Mechkov, S., \& Sausset, F. 2008, EL, 81, 44002

Schulz, R., Hilchenbach, M., Langevin, Y., et al. 2015, Natur, 518, 216

Skorov, Y., \& Blum, J. 2012, Icar, 221, 1

Stephan, T. 2008, SSRv, 138, 247

Stephan, T., Rost, D., Vicenzi, E. P., et al. 2008, M\&PS, 43, 233

Stodolna, J., Gainsforth, Z., Butterworth, A. L., \& Westphal, A. J. 2014 E\&PSL, 388, 367

Tuzzolino, A. J., Economou, T. E., Clark, B. C., et al. 2004, Sci, 304, 1776

Vinogradoff, V., Fray, N., Duvernay, F., et al. 2013, A\&A, 551, A128

Zolensky, M. E., Zega, T. J., Yano, H., et al. 2006, Sci, 314, 1735 